\section{Optimal Trajectories for Maximum Endurance Gliding in a Horizontal Plane}

\author{
Nguyen Xuan Vinh* \\ University of Michigan, Ann Arbor, Michigan \\ and \\ Ching-Yew Yang $\dagger$ and Jeng-Shing Chern $\ddagger$
}

Chung Shan Institute of Science and Technology

Lungtan, Taiwan, Republic of China

\begin{tabular}{|c|c|}
\hline \multirow{2}{*}{\multicolumn{2}{|c|}{ Nomenclature }} \\
\hline & \\
\hline$C_{0}, C_{1}, C_{2}, C_{3}$ & $=$ constants of integration \\
\hline$C_{D}$ & $=$ drag coefficient \\
\hline$C_{D o}$ & $=$ zero lift drag coefficient \\
\hline$C_{L}$ & $=$ lift coefficient \\
\hline$C_{L}^{*}$ & $\begin{aligned}= & \text { lift coefficient for maximum lift-to-drag } \\
& \text { ratio }\end{aligned}$ \\
\hline$D$ & $=\mathrm{drag}$ force \\
\hline$E^{*}$ & $=$ maximum lift-to-drag ratio \\
\hline$h$ & $=$ altitude \\
\hline$H$ & $=$ Hamiltonian function \\
\hline$k_{1}, k_{2}, k_{3}$ & $\begin{aligned}= & \text { constants, }=C_{1} / C_{0}, C_{2} / C_{0}, C_{3} / C_{0},\end{aligned}$ \\
\hline$K$ & $=$ induced drag factor \\
\hline$L$ & $=$ lift force \\
\hline$m$ & $=$ mass of the vehicle \\
\hline$p_{x}, p_{y}, p_{u}, p_{\psi}, p_{\theta}$ & $\begin{aligned}= & \text { adjoint variables associated with state } \\
& \text { variables }\end{aligned}$ \\
\hline$r$ & $=$ radius of penetration \\
\hline$S$ & $=$ reference area \\
\hline$t$ & $=$ time \\
\hline$u$ & $=$ dimensionless speed \\
\hline$V$ & $=$ speed of vehicle \\
\hline$W$ & $=$ weight of vehicle \\
\hline$x, y$ & $=$ dimensionless coordinates \\
\hline$X, Y$ & $=$ position coordinates of vehicle \\
\hline$\Delta$ & $=\tan \mu$ \\
\hline$\theta$ & $=$ dimensionless time \\
\hline$\lambda$ & $=$ normalized lift coefficient \\
\hline$\mu$ & $=$ bank angle \\
\hline$\rho$ & $=$ density of atmosphere \\
\hline$\tau$ & $=$ normalized time \\
\hline$\psi$ & $=$ velocity yaw angle \\
\hline$\omega$ & $=$ dimensionless wing loading \\
\hline Subscripts & \\
\hline 0 & $=$ initial conditions \\
\hline$f$ & $=$ final conditions \\
\hline
\end{tabular}

\section{Introduction}

$\Gamma$ HE problem of determining minimum and maximum endurance trajectories for subsonic gliding flight in a horizontal plane has been considered previously. ${ }^{1,2}$ The control variable was parametrized as a polynomial of the normalized time; hence, the problem becomes a parameter optimization problem. In both references, a specified small lifting vehicle, which can be used as a low-level weapons delivery system, is used for the numerical integration. This

Presented as Paper 81-1868 at the AIAA Atmospheric Flight Mechanics Conference, Albuquerque, N. Mex., Aug. 19-21, 1981; submitted Sept. 30, 1981; revision received June 6, 1983. Copyright (C) American Institute of Aeronautics and Astronautics, Inc., 1983. All rights reserved.

*Professor, Aerospace Engineering.

† Senior Scientist. Member AIAA.

$\ddagger$ Associate Scientist. Member AIAA.
Note presents the optimal solutions to the maximum endurance trajectories by the application of Pontryagin's maximum principle. ${ }^{3,4}$ Furthermore, by the use of a set of dimensionless variables, the solutions obtained are applicable to a whole class of glide vehicles.

\section{Variational Formulation}

The geometry of gliding flight in a horizontal plane is depicted in Ref. 1 and the motion is governed by the state equations $^{5}$

$$
\begin{aligned}
\dot{X} & =V \cos \psi \\
\dot{Y} & =V \sin \psi \\
\dot{V} & =-D / m \\
\dot{\psi} & =L \sin \mu / m V \\
L \cos \mu & =W
\end{aligned}
$$

We shall consider a parabolic drag polar of the form

$$
C_{D}=C_{D o}+K C_{L}^{2}
$$

where, at subsonic speeds, $C_{D O}$ and $K$ are considered constant. By the use of the dimensionless system

$$
\begin{gathered}
x=g X / V_{0}^{2}, \quad y=g Y / V_{0}^{2}, \quad u=V / V_{0}, \quad \theta=g t / V_{0} \\
\omega=2 W / \rho S V_{0}^{2} C_{L}^{*}
\end{gathered}
$$

we obtain the set of dimensionless equations of motion ${ }^{4}$

$$
\begin{aligned}
& x^{\prime}=u \cos \psi \\
& y^{\prime}=u \sin \psi \\
& u^{\prime}=-\left(u^{2} / 2 E^{*} \omega\right)\left[1+\left(\omega^{2} / u^{4} \cos ^{2} \mu\right)\right] \\
& \psi^{\prime}=\tan \mu / u \\
& \theta^{\prime}=I
\end{aligned}
$$

where the prime denotes the derivative taken with respect to the dimensionless time $\theta$. In this system, the only performance parameter involved is the maximum lift-to-drag ratio $E^{*}$. The dimensionless wing loading, $\omega$, which is a constant in level flight is a physical characteristic for a whole class of glide vehicles. It can be used to analyze the influence of the altitude on gliding performance. ${ }^{6}$ The constraining equation, Eq. (1e), becomes, in this formulation,

$$
\cos \mu=\omega / \lambda u^{2}
$$

where $\lambda$ is the normalized lift coefficient

$$
\lambda=C_{L} / C_{L}^{*}
$$

Because of this constraint, the bank angle $\mu$ is the sole control in the dynamical system. In turning flight, there are two physical constraints on the maneuverability. The first one is the load factor

$$
n=L / W=1 / \cos \mu
$$

This value is bounded by an upper limit $n=n_{\max }$, which is a physiological/structural limit. The other constraint is the maximum lift coefficient $C_{L \max }$, or in normalized form, $\lambda_{\max }$. Hence, the bank control belongs to the set

$$
0 \leq|\mu| \leq \inf \left[\cos ^{-1}\left(1 / n_{\max }\right), \cos ^{-1}\left(\omega / u^{2} \lambda_{\max }\right)\right]
$$


Using the maximum principle, we introduce the adjoint vector $\boldsymbol{p}$ to form the Hamiltonian

$$
\begin{aligned}
H & =p_{x} u \cos \psi+p_{y} u \sin \psi-p_{u}\left(u^{2} / 2 E^{*} \omega\right)\left[I+\left(\omega^{2} / u^{4} \cos ^{2} \mu\right)\right] \\
& +p_{\psi}(\tan \mu / u)+p_{\theta}
\end{aligned}
$$

It is known that the variational problem has the integrals ${ }^{4}$

$$
\begin{aligned}
& p_{\theta}=C_{0} \\
& p_{x}=C_{1} \\
& p_{y}=C_{2} \\
& p_{\psi}=C_{1} y-C_{2} x+C_{3}
\end{aligned}
$$

Furthermore, since the time is an ignorable coordinate, and in the maximum endurance problem the final time is not specified, we have identically for the whole duration of the flight $H=0$. For interior bank control, we have $\partial H / \partial \mu=0$, or

$$
\tan \mu=\left(\frac{p_{\psi}}{p_{u}}\right)\left(\frac{E^{*} u}{\omega}\right)
$$

Using the integrals, Eqs. (10), and $H=0$ in the optimal relation, Eq. (11), we have the equation

$$
\begin{gathered}
\left(k_{1} y-k_{2} x+k_{3}\right) \Delta^{2}+2 u\left[1+u\left(k_{l} \cos \psi+k_{2} \sin \psi\right)\right] \Delta \\
-\frac{\left(k_{l} y-k_{2} x+k_{3}\right)}{\omega^{2}}\left(\omega^{2}+u^{4}\right)=0
\end{gathered}
$$

where $\Delta=\tan \mu$.

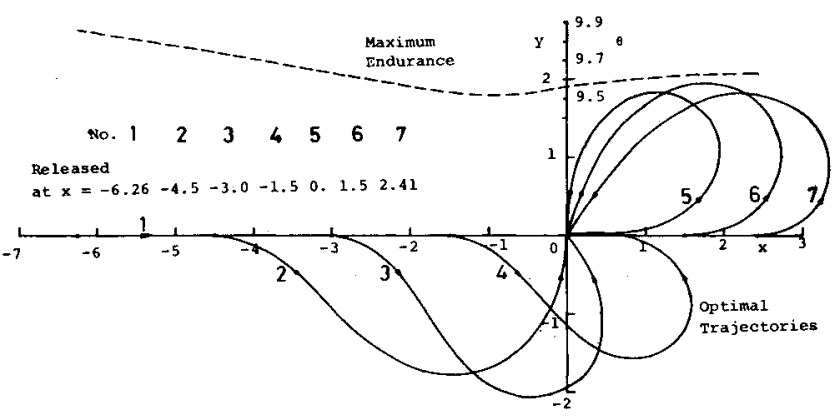

Fig. 1 Optimal trajectories and maximum endurances.

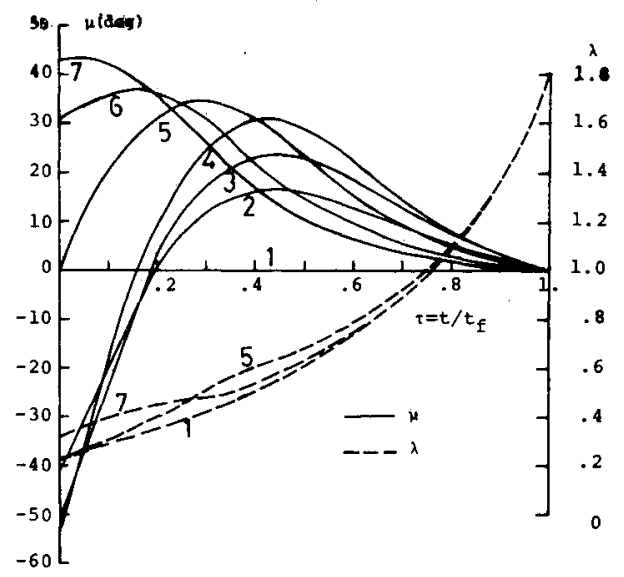

Fig. 2 Variations of $\mu$ and $\lambda$ for the optimal trajectories.

\section{Maximum Endurance Problems}

As an application, it is supposed that a small lifting vehicle is carried by an airplane heading directly toward a target location at the origin $\mathcal{O}$. By symmetry, we can consider the initial velocity as along the $X$ axis. At a certain distance, the gliding vehicle is released and, while the carrier is performing an escaping maneuver, the small vehicle tries to reach the target at the final residual speed $u_{f}$ in maximum time. Thus, we have the end conditions

$$
\begin{aligned}
& \theta_{0}=0, \quad x_{0}=\text { specified }, \quad y_{0}=\psi_{0}=0, \quad u_{0}=1 \\
& \theta_{f}=\max , \quad x_{f}=y_{f}=0, \quad \psi_{f}=\text { free }, \quad u_{f}=\text { specified }
\end{aligned}
$$

For the numerical integration, we take the values

$$
E^{*}=20, \quad \lambda_{\max }=1.8, \quad \omega=0.23
$$

The first two values concern the aerodynamic characteristics of the gliding vehicle. They represent typical values for a whole class of vehicles, which includes the example vehicle in Refs. 1 and 2. The value of $\omega$ corresponds to the same vehicle at sea level, but it can represent a vehicle with lower wing loading flying at higher altitude. To enforce the constraint on the load factor, we can specify a value, say, $n_{\max }=5$. But, in general, for maximum endurance problems this value is never reached since the bank angle remains in the interior of the domain of maneuverability.

For the small glider to reach the target, it must be released within a zone of penetration. This zone has a limiting radius $r$ which can be obtained by integrating the state equations with $\mu=\psi=0$. The solution is

$$
r=\left|x_{\max }\right|=\frac{E^{*}}{2} \omega \log \left[\frac{\lambda_{\max }^{2}}{1+\lambda_{\max }^{2}}\left(1+\frac{l}{\omega^{2}}\right)\right]
$$

with an absolute maximum endurance

$$
\begin{aligned}
\theta_{\max } & =\sqrt{\frac{\omega}{2}} E^{*}\left[\tan ^{-1}\left(\frac{\sqrt{2 \lambda_{\max }}}{1-\lambda_{\max }}\right)-\tan ^{-1}\left(\frac{\sqrt{2 \omega}}{1-\omega}\right)\right. \\
+ & \left.\frac{1}{2} \log \left(\frac{\lambda_{\max }+\sqrt{2 \lambda_{\max }}+1}{\lambda_{\max }-\sqrt{2 \lambda_{\max }}+1}\right)-\frac{1}{2} \log \left(\frac{\omega+\sqrt{2} \omega+1}{\omega-\sqrt{2} \omega+1}\right)\right]
\end{aligned}
$$

In deriving these explicit limiting solutions, the final speed is the stall speed which corresponds to maximum lift coefficient at zero bank angle. Hence from Eq. (5),

$$
u_{f}=\sqrt{\omega / \lambda_{\max }}
$$

With free $\psi_{f}, p_{\psi}\left(\theta_{f}\right)=0$, and from Eq. $(10 \mathrm{~d}), k_{3}=0$. Hence, the optimal problem is a two-parameter problem, with $k_{l}$ and $k_{2}$ to be selected such that at $u=u_{f}$ the final conditions $x_{f}=y_{f}=0$ are identically satisfied. The five optimal trajectories with points of release before and directly above the target are shown in Fig. 1. The maximum endurances for different points of release are also given in Fig. 1. The plot of the variations of the bank angle and the normalized lift coefficient $\lambda$ vs the normalized time $\tau=t / t_{f}$ is shown in Fig. 2 .

Figure 1 also presents two optimal trajectories at different points of release while the carrier is leaving the target. The last trajectory is the limiting trajectory for the released vehicle to have sufficient speed to reach the target. This trajectory can be obtained directly by translating the origin of coordinates to the point of release and solving the problem with the end conditions

$$
\begin{array}{lll}
\theta_{0}=0, \quad x_{0}=y_{0}=0, & \psi_{0}=0, & u_{0}=1 \\
\theta_{f}=\text { max }, \quad x_{f}=\text { free }, & y_{f}=0, & \psi_{f}=\text { free } \\
u_{f}=\text { specified } & &
\end{array}
$$


In this case, the optimal bank angle is still given by Eq. (12), but now with $k_{l}=0, \mathrm{k}_{3}=\mathrm{k}_{2} \mathrm{x}_{\mathrm{f}}$. Hence, the optimal bank angle is given by

$k_{2}\left(x_{f}-x\right) \Delta^{2}+2 u\left[I+k_{2} u \sin \psi\right] \Delta-\frac{k_{2}\left(x_{f}-x\right)}{\omega^{2}}\left(\omega^{2}+u^{4}\right)=0$

The parameters selected for the iteration are $k_{2}$ and $x_{f}$.

\section{References}

!Williamson, W.E., "Minimum and Maximum Endurance Trajectories for Gliding Flight in a Horizontal Plane," Journal of Guidance and Control, Vol. 2, Nov.-Dec. 1979, pp. 457-463.

${ }^{2}$ Williamson, W.E., "Maximum-Endurance Return-to-Target Gliding Trajectories," Journal of Guidance and Control, Vol. 3, Sept.-Oct. 1980, pp. 474-477.

${ }^{3}$ Bryson, A.E. and Ho, Y.C., Applied Optimal Control, Hemisphere Publishing Corp., Washington, D.C., 1975.

${ }^{4}$ Vinh, N.X., Optimal Trajectories in Atmospheric Flight, Elsevier Scientific Publishing Co., Amsterdam, 1981.

${ }^{5}$ Miele, A., Flight Mechanics 1: Theory of Flight Paths, AddisonWesley, Reading, Mass., 1962.

${ }^{6}$ Busemann, A., Vinh, N.X., and Culp, R.D., "Optimum Altitude for Coasting Flight of a Hypervelocity Vehicle," Journal of the Astronautical Sciences, Vol. 21, July-Aug. 1973, pp. 32-48.

\section{A Model Reference Adaptive Control Algorithm Applied to Terminally Controlled Systems}

\author{
Hagop Panossian* \\ HR Textron Inc., Irvine, California
}

\section{Introduction}

$\mathbf{F}$ OR the past few decades, programs directed toward enhancing the air-to-ground weapon delivery accuracy of aircraft have been undertaken by the U.S. Air Force and Navy. These programs have been basically aimed at optimizing the handling qualities of the aircraft's weapon delivery and fire control systems. The underlying strategy has been to minimize the errors at release. Efforts to compensate for release mechanism errors have been pursued through analytical means. ${ }^{1,2}$

The main contributing factors in air-to-ground bombing dispersion are the following: positioning errors, weapon release mechanism errors, separation disturbances, bomb anomalies, wind gusts, and pilot-induced errors. It is possible, by various means, to reduce some of these errors. However, most of the dispersion will still exist under most realistic weapon release conditions. Even though laser-, infrared-, and television-guided bombs have solved a large portion of the dispersion problem, the various target acquisition and designation constraints involved in hitting a target renders their use very costly and sometimes impractical.

Missile guidance is enhanced by the movement of control surfaces that produce a response in the form of an acceleration. An adaptive scheme can be used to compare the

Received Jan. 5, 1983; revision received June 9, 1983. Copyright (C) American Institute of Aeronautics and Astronautics, Inc., 1983. All rights reserved.

${ }^{*}$ Senior Analytical Engineer. Member AIAA. actual vs the desired outputs. ${ }^{3,4}$ Model reference adaptive control is very suitable for ballistic air-to-ground missiles since a predetermined trajectory is normally to be followed. ${ }^{5}$ It should be underlined that adaptive controllers will try to keep the bomb dynamics relatively invariant with respect to the dynamic pressure.

\section{Problem Statement}

Consider a bomb released from an aircraft under "ideal" conditions, with no perturbations whatsoever and no pre- or postrelease errors. Then, the bomb will follow a fixed trajectory for a given initial velocity and attitude. The aerodynamic equations of a bomb in a ballistic trajectory are given (in twodimensional space for convenience) by

$$
\begin{aligned}
& \dot{v}=(1 / m)(-D-m g \sin \gamma) \\
& \dot{\gamma}=(1 / m v)(L-m g \cos \gamma)
\end{aligned}
$$

where $v$ is the velocity, $m$ the mass, $D$ the total drag, $g$ the acceleration due to gravity, $\gamma$ the flight path angle, $(\cdot)$ the time derivative, and $L$ the lift.

$$
\begin{aligned}
& \ddot{\theta}=(1 / \mathrm{J})\left(-\mathrm{M}_{\dot{\theta}} \dot{\theta}-M_{\alpha} \dot{\alpha}\right) \\
& \dot{\alpha}=\dot{\theta}-\dot{\gamma}
\end{aligned}
$$

where $\theta$ is the pitch angle, $J$ the moment of inertia, $M_{\theta}$ and $M_{\alpha}$ the moment coefficients, and $\alpha$ the angle of attack.

The main cause of deviation of a bomb from a nominal ballistic trajectory, as defined above, is an oscillatory angle of attack primarily induced during release and separation from the aircraft by aerodynamic moments, by mechanical torques imparted by the ejection and release mechanism, and by mass variations. Such effects can be modeled as changes in the velocity components, in the mass, and in the attitude.

To formulate the problem of hitting a target with a guided bomb, the following assumptions were made:

1) There are onboard accelerometers and rate gyros that will furnish the quantities required to determine accurately the state of the bomb at given instants.

2) Control fins placed at the tail end of the bomb are moved, as required, by actuators in the tail cone.

The equations of motion with perturbations and the contributions due to the control surfaces can now be represented in the following manner:

$$
\begin{aligned}
& \dot{v}=\frac{I}{m}\left[-D-\Delta D-(m+\Delta m) g \sin (\gamma+\Delta \gamma)-D_{c}\right] \\
& \dot{\gamma}=\frac{1}{m v}\left[L+\Delta L-(m+\Delta m) g \cos (\gamma+\Delta \gamma)+L_{c}\right] \\
& \ddot{\theta}=(I / J)\left(M+\Delta M+M_{c}\right) \\
& \dot{\alpha}=\dot{\theta}+\Delta \dot{\theta}+\dot{\theta}_{c}-\left(\dot{\gamma}+\Delta \dot{\gamma}+\dot{\gamma}_{c}\right)
\end{aligned}
$$

where the $\Delta$ indicate the random incremental changes that vary a few times ( $\Delta m$ varies only once) during a trajectory and have Gaussian distributions. Subscript $c$ stands for the contribution due to the controls.

Now, with the initial conditions and the respective random perturbations appropriately quantified, it is not difficult to solve the ballistic trajectory of a bomb (either with or without perturbations) by means of an Adams-Moulton integration algorithm with a Runge-Kutta startup. The problem is to guide the bomb to the nominal trajectory by utilizing the measurements (assumed exact) from the available sensors and to perform the necessary corrections by deflecting the fins an appropriate amount from the release point down to impact. 